\title{
Schutzschild nach außen, Antreiber nach innen
}

\author{
Martin Beck
}

\begin{abstract}
Aufsichtsräte wirken meist im Verborgenen und haben doch vielfach großen Einfluss auf die Unternehmenspolitik sozialer Organisationen. In intensiven Gesprächen gewährten drei Aufsichtsräte aus der Sozialwirtschaft detaillierte Einblicke in ihre Tätigkeit.
\end{abstract}

Aufsichtsräte sind selten in der Öffentlichkeit zu sehen. Sie repräsentieren nicht, sie führen nicht an, sie treten nicht auf, sie werden nicht gefeiert. Die Bühne gehört den Vorständen und den Geschäftsführungen. Daraus sollte man nicht schließen, Aufsichtsräte hätten nichts zu sagen. Ganz im Gegenteil: Ihre Position hat sich innerhalb weniger Jahre verändert. Sie wurden durch den Gesetzgeber gestärkt, ihre Aufgabe erhielt durch die Corporate Governance Bewegung ein schärferes Profil und sie gewinnen landauf landab an Selbstbewusstsein. Dieses neue Selbstbewusstsein lässt sich zum Beispiel an der zunehmenden Zahl überraschender Abgänge von Sozialmanagern ablesen, aber nicht nur daran.

Es ist noch keine halbe Generation her, da erwartete man von den Aufsichtsgremien sozialwirtschaftlicher Unternehmen in erster Linie, dass sie die begeisternden Aktionen und Aktivitäten der hauptamtlichen Führung ebenso begeisternd fanden und alles dafür taten, um die Spielräume der Stars möglichst gut bespielbar zu machen und zu halten. Auch heute noch verbergen viele Sozialunternehmen in ihren Internetauftritten und auf ihren Briefbögen schamhaft den Namen des oder der Vorsitzenden des Aufsichtsgremiums, als ob man darüber besser nicht sprechen sollte. Andere Werke gehen einen anderen Weg und holen ihre Aufsichtsräte, jedenfalls fallweise, mit auf die Bühne. Das kann taktische Gründe haben (der Vorstand will sich hinter dem hoch renommierten Aufsichtsratsvorsitzenden verstecken) oder es ist Klugheit (es kostet wenig, dem Aufsichtsrat etwas vom Rampenlicht abzugeben, aber es sieht gut aus und es wirkt meist positiv).

Wer so viel Macht hat - vor allem Macht über die obersten Führungspersonen, aber auch über die wichtigsten Finanzentscheidungen - und zugleich fast im Unsichtbaren wirkt, der macht neugierig. Neugierig darauf, welche Menschen solche Ämter übernehmen, wie viel Zeit sie investieren, ob sie das für Geld oder für Ruhm oder aus einer anderen persönlichen Motivation heraus machen oder was ihre Antriebskräfte sind.

Weil in der Sozialwirtschaft eine deutliche Tendenz zum Größenwachstum - meist durch Fusionen oder Über-

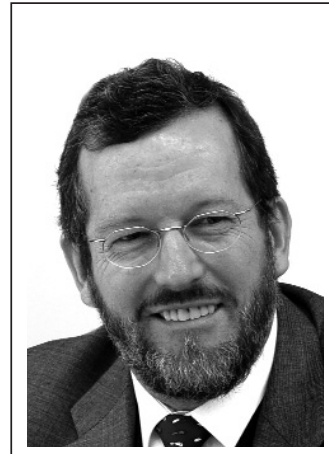

Prof. Martin Beck (57), Großhandelskaufmann und Diplombetriebswirt (FH), war Geschäftsführer im Diakonischen Werk Württemberg, Gründer und Geschäftsführer der Beratungsgesellschaft BSU und Sanierer in der Industrie. Er ist jetzt selbstständiger Unternehmensberater, Autor, Aufsichtsrat und Hochschullehrer. Internet http://www.prof-beck.net nahmen, weniger aus dem Geschäft heraus - sichtbar ist, hat der Autor die ehrenamtlichen Vorsitzenden von drei großen, teils regional, teils national, teils international tätigen, Sozialunternehmen besucht und sie nach ihren Motiven und ihrer Führungspraxis befragt. Vertreten sind Caritas, Diakonie und Parität. Jeder der drei Herren (das ist die Regel; dem Autor ist nur eine Dame als Vorsitzende eines der größeren Sozialwerke bekannt) verantwortet einen sozialwirtschaftlichen Geschäftsumfang in dreistelliger Millionenhöhe, in zwei Fällen rückt die halbe Milliarde in Sichtweite.

Wer sind eigentlich die Leute, die unsere mächtigen Sozialmanager heuern und feuern? Was treibt sie an, was reizt sie, was beschwert sie? Welche persönlichen und beruflichen Voraussetzungen müssen gegeben sein, damit man ein solches zeitaufwendiges Ehrenamt annehmen und ausfüllen kann?

Alle drei befragten Gesprächspartner waren übereinstimmend der Meinung, dass es trotz erhöhter Qualifikationsanforderungen nicht schwerer geworden sei, Gremienmitglieder zu finden. Für eine interessante Aufgabe finde man immer gute Leute, war die einhellige Meinung. Im Umkehrschluss lässt sich dann wohl sagen, dass dort, wo man sich mit der Gewinnung von Nachwuchs und Nachfolgern in den Gremien schwer tut, offensichtlich mehr der Grauschleier als der Glanz dominieren. Ein Grund also, selbstkritisch in sich zu gehen und kritische Fragen an das eigene Werk und die eigene Arbeit zu stellen.

Und schließlich: Gibt es so etwas wie eine Typologie der Aufsichtsratsvorsitzenden? Ja, das gibt es offensicht- 
lich. Hier ein vorsichtiger Versuch einer Typisierung von Vorsitzenden:

- Mann

- ab 50 Jahren aufwärts mit 20 bis 30 absolvierten Berufsjahren

- außerhalb des Ehrenamtes: berufliche Führungsposition, politisches Wahlamt oder erfolgreiche Selbstständigkeit

- wirtschaftlich unabhängig, was entweder durch ein gesichertes Einkommen oder eine angenehme Pension oder aber ein erfolgreiches Handeln als Unternehmer gewährleistet wird

- relative Freiheit der Zeitgestaltung (aber bitte nicht so viel, dass ein »Übervorstand « entsteht)

- ökonomische Unabhängigkeit (also keine akute Versuchung, aus dem Ehrenamt Kapital zu schlagen)

- braucht das Ehrenamt nicht, um seinen sozialen Wunschstatus zu erreichen, sondern kann seinen bereits erreichten Status zugunsten des Amtes einsetzen (Er «ist schon etwas«)

- aus der Mitte des Gremiums gewählt (erfolgreiche Nachwuchsarbeit)

Wenn man ein solches Amt ernsthaft und verantwortungsvoll ausüben will, muss man - neben vielen anderen Voraussetzungen - vor allem Zeit mitbringen. Nicht alle Zeit der Welt - schließlich wollen die Vorstände auch einmal in Ruhe ihre Arbeit machen. Aber doch so viel Zeit, dass neben den pflichtgemäß zu absolvierenden Sitzungen auch regelmäßige Gespräche mit dem Vorstand möglich sind, dass auch die eine oder andere repräsentative Verpflichtung möglich ist und dass der Vorstand weiß, dass er im politischen Ernstfall, wenn Verhandlungen zu scheitern drohen, auf den Vorsitzenden des Aufsichtsrates zurückgreifen kann.

Wer über ein solches beneidenswertes Zeitbudget verfügt, der ist entweder Pensionär (nicht schlecht, aber mit einem gewissen Verfallsdatum versehen), oder von Beruf Erbe (auch nicht schlecht, weil das auch die Chance zur einen oder anderen finanziellen Zuwendung an das Werk möglich machen könnte) oder aktiver und erfolgreicher Geschäftsmann (sehr gut, aber meist unter extremem Zeitdruck) oder leitender Beamter oder Kirchenführer mit sicherem Einkommen und deshalb ohne Erwerbsdruck. Der Blick wird also auf den gehobenen Mittelstand oder das Bildungs- und Vermögensbürgertum fallen, wenn für ein großes Werk ein erfahrener Vorsitzender gesucht wird.

Und, fragt man sich: Gibt es auf dem Gebiet der Aufsichtsräte spürbare Unterschiede zwischen den Wohlfahrtsverbänden? Nein, nach allem, was der Autor weiß und beobachtet, gibt es wohl keine großen Unterschiede über die Verbandsgrenzen hinweg. Die Ähnlichkeiten sind sogar frappierend. Alle brauchen sie unabhängige Vorsitzende, also Menschen, die nicht nur Zeit haben, sondern die auch Erfahrung in der Führung von Organi- sationen verfügen - und die charakterlich stark genug sind, ihre eigene Führungserfahrung so weit zurückzunehmen, dass wirkungsvolle Aufsicht möglich ist. Es ist vielleicht nicht zufällig, dass zwei der drei Gesprächspartner am Ende einer erfolgreichen politischen Karriere ein solches Ehrenamt übernahmen.

Bei aller Individualität der Verbände und ihrer Mitgliedseinrichtungen lassen sich aber doch Erfahrungswerte formulieren, bei deren Anwendung die Zusammenarbeit zwischen Aufsichtsorgan und Vorstand wirksam sein kann. Ein guter Aufsichtsrat, so könnte man zugespitzt formulieren, ist Schutzschild nach außen und Antreiber nach innen, er hält dem Vorstand den Rücken frei, aber er sagt ihm auch deutlich Bescheid, wenn die Dinge nicht so laufen, wie das besprochen und beschlossen war. Offenheit, Diskurs und, wenn es denn sein muss, ernsthafte Auseinandersetzung nach innen - und zugleich höchste Loyalität nach außen; so könnte eine ideale Aufgabenund Rollenverteilung beschrieben werden. Hier eine erfahrungsgesättigte und praxiserprobte Gebrauchsanweisung für eine funktionierende Zusammenarbeit von Aufsicht und Führung:

- kleines Gremium (3 bis 6 Personen)

- respektvolle Distanz zwischen Aufsichtsrat und Vorstand statt distanzloser Kameraderie

- innere Unabhängigkeit der Aufseher vom Unternehmen und von Personen

- Vorschussvertrauen auf der Basis von klaren Regelungen

- ausreichende Spielräume für das Tun und Lassen der Vorstände

- klares Berichtswesen auf der Basis von vereinbarten Regelungen

- regelmäßige Gespräche zwischen dem Aufsichtsratsvorsitzenden und den Vorständen

- Sicherheit über Hol- und Bringschulden des Vorstands gegenüber dem Aufsichtsgremium

- aktiv ausgeübte Fürsorgepflicht durch den/die Vorsitzende(n) des Aufsichtsorgans

- aufmerksame Beobachtung des Leitungshandelns durch den Aufsichtsrat

- Lob (öffentlich) und Tadel (diskret) durch den Aufsichtsrat

- Vertraulichkeit und Diskretion über alles, was die Angelegenheiten der Vorstände betrifft

- selbstkritische Haltung des Aufsichtsgremiums bezüglich der eigenen Wirkung

- Verzicht auf Repräsentativität von Berufen und Interessengruppen

- Gewicht auf Lebens- und Berufserfahrung (weiter S. 20) 


\begin{tabular}{|c|c|c|c|}
\hline & Erich Schneider & Dr. Joachim Senn & Gerd Weimer \\
\hline Jahrgang & 1933 & 1954 & 1948 \\
\hline Berufsweg & $\begin{array}{l}\text { Verwaltungsbeamter, 1960-1979 Bür- } \\
\text { germeister der Gemeinde Burgstall } \\
\text { (später Burgstetten) Rems-Murr-Kreis. } \\
\text { 1968-1992 Abgeordneter im Landtag } \\
\text { von Baden-Württemberg (CDU), ab } \\
1976 \text { stellvertretender Fraktionsvorsit- } \\
\text { zender, ab } 1982 \text { Landtagspräsident. }\end{array}$ & $\begin{array}{l}\text { Studium der Betriebswirtschaftslehre } \\
\text { in München; Abschluss Diplom-Kauf- } \\
\text { mann. Promotion zum Dr. rer.pol. in } \\
\text { München. } \\
\text { Geschäftsführender Gesellschafter der } \\
\text { Lorenz Senn GmbH \& Co KG, die Betei- } \\
\text { ligungen an Medienunternehmen hält } \\
\text { (z.B. Bodensee-Medienzentrum, Tett- }\end{array}$ & $\begin{array}{l}\text { Gymnasiallehrer (Politik, Geographie, } \\
\text { Sport). } \\
1984 \text { - } 2001 \text { Abgeordneter im Landtag } \\
\text { von Baden-Württemberg (SPD) } \\
1998 \text { - } 2006 \text { Erster Bürgermeister der } \\
\text { Stadt Tübingen }\end{array}$ \\
\hline $\begin{array}{l}\text { Aufsichtsratsvorsitzender bei } \\
\ldots\end{array}$ & $\begin{array}{l}\text { Christliches Jugenddorfwerk Deutsch- } \\
\text { lands e. V. (cjd), Ebersbach/Fils } \\
\text { (Satzungsmäßige Bezeichnung: } \\
\text { Präsident). }\end{array}$ & $\begin{array}{l}\text { Stiftung Liebenau, Meckenbeuren } \\
\text { (Baden-Württemberg). }\end{array}$ & $\begin{array}{l}\text { Paritätischer Wohlfahrtsverband Ba- } \\
\text { den-Württemberg e.V., Stuttgart (Vor- } \\
\text { standsvorsitzender). } \\
\text { Baden-Württembergischer Landesver- } \\
\text { band für Prävention und Rehabilitation, } \\
\text { Renchen (Aufsichtsratsvorsitzender). }\end{array}$ \\
\hline $\begin{array}{l}\text { Zugehörigkeit zu einem } \\
\text { Wohlfahrtsverband }\end{array}$ & Diakonisches Werk. & Caritasverband. & Paritätischer Wohlfahrtsverband. \\
\hline Das Unternehmen & $\begin{array}{l}\text { Das Christliche Jugenddorfwerk } \\
\text { Deutschland e. V. (CJD) wurde } 1947 \\
\text { von Pfarrer Arnold Dannenmann ge- } \\
\text { gründet. Es ist heute mit mehr als } 100 \\
\text { Einrichtungen und einem Ge- } \\
\text { schäftsumfang von mehr als } 400 \text { Mio. } \\
€ \text { der größte Jugendhilfeträger und ei- } \\
\text { nes der größten Sozialunternehmen } \\
\text { Deutschlands. }\end{array}$ & $\begin{array}{l}\text { Die Stiftung Liebenau wurde } 1866 \\
\text { (Stiftung: 1868) von Kaplan Adolf Aich } \\
\text { aus Tettnang zusammen mit einigen } \\
\text { Bürgern der Stadt gegründet. Sie ist } \\
\text { heute das Dach eines weit verzweigten } \\
\text { Sozialkonzerns mit einem guten Dut- } \\
\text { zend Tochter- und Beteiligungsgesell- } \\
\text { schaften im In- und Ausland und einem } \\
\text { Geschäftsumfang von mehr als } 200 \\
\text { Mio. €. }\end{array}$ & $\begin{array}{l}\text { Der Landesverband Baden-Württem- } \\
\text { berg des Paritätischen ist einerseits } \\
\text { Wohlfahrtsverband. Über eine Beteili- } \\
\text { gungsgesellschaft ist er an fast } 30 \text { Or- } \\
\text { ganisationen und Unternehmen betei- } \\
\text { ligt. } \\
\text { Der Baden-Württembergische Landes- } \\
\text { verband für Prävention und Rehabilitati- } \\
\text { on entstand aus dem Zusammen- } \\
\text { schluss der Drogenhilfe (Tübingen) und } \\
\text { des Badischen Landesverbandes gegen } \\
\text { die Suchtgefahren (Renchen) und ist } \\
\text { heute der größte Anbieter von Suchthil- }\end{array}$ \\
\hline Mitglied dieses Gremiums seit & 1993. & 1990. & $\begin{array}{l}\text { Paritätischer Baden-Württemberg seit } \\
2005 . \\
\text { Drogenhilfe/ Der Baden-Württembergi- } \\
\text { sche Landesverband für Prävention } \\
\text { und Rehabilitation seit } 1997\end{array}$ \\
\hline $\begin{array}{l}\text { Vorsitzender des Gremiums } \\
\text { seit }\end{array}$ & $\begin{array}{l}1993 \text { (bis } 2000 \text { als Vorstand, seither, } \\
\text { nach einer Satzungsänderung, als Auf- } \\
\text { sichtsrat) }\end{array}$ & $\begin{array}{l}\text { Seit } 1993 \text { stellvertretender Vorsitzen- } \\
\text { der, } 1996 \text { Vorsitzender. }\end{array}$ & $\begin{array}{l}\text { Paritätischer Baden-Württemberg: } \\
2005 . \\
\text { Drogenhilfe/ Der Baden-Württembergi- } \\
\text { sche Landesverband für Prävention }\end{array}$ \\
\hline $\begin{array}{l}\text { Wie sind Sie damals gefunden } \\
\text { und gewonnen worden? }\end{array}$ & $\begin{array}{l}\text { Angesprochen und angeworben von } \\
\text { den heutigen Vizepräsidenten des Wer- } \\
\text { kes. Man wollte die als Bürgermeister, } \\
\text { Bankaufsichtsrat und Landespolitiker } \\
\text { gewonnenen Erfahrungen und Netz- } \\
\text { werke nutzen. }\end{array}$ & $\begin{array}{l}\text { Man wollte den Altersschnitt senken. } \\
\text { Auswahlkriterien waren unter anderem } \\
\text { wirtschaftliche und unternehmerische } \\
\text { Erfahrung und regionaler Bezug. }\end{array}$ & $\begin{array}{l}\text { Paritätischer Wohlfahrtsverband: als } \\
\text { Folge des politischen Engagements. } \\
\text { Drogenhilfe/Der Baden-Württembergi- } \\
\text { sche Landesverband für Prävention } \\
\text { und Rehabilitation: aus der kommuna- } \\
\text { len Rolle als Bürgermeister heraus. }\end{array}$ \\
\hline
\end{tabular}




\begin{tabular}{|c|c|c|c|}
\hline & Erich Schneider & Dr. Joachim Senn & Gerd Weimer \\
\hline $\begin{array}{l}\text { Was bedeutet Ihnen diese Auf- } \\
\text { gabe heute? }\end{array}$ & $\begin{array}{l}\text { Es ist eine große Verpflichtung, als Ge- } \\
\text { genüber des zweiköpfigen Vorstandes } \\
\text { mit zur Wirksamkeit und zum Erfolg } \\
\text { des Werkes beizutragen. }\end{array}$ & $\begin{array}{l}\text { Tätigkeit ist mit vielen positiven Aspekten } \\
\text { und Erfahrungen verbunden. Interessant } \\
\text { das Zusammenbringen von Fachlichkeit, } \\
\text { sozialem Engagement und Wirtschaftlich- } \\
\text { keit, aber auch die Kontakte zu Mitarbeitern }\end{array}$ & $\begin{array}{l}\text { Beide Aufgaben sind sinnstiftend. Die } \\
\text { Freiheit des Ehrenamtes ist reizvoll. }\end{array}$ \\
\hline $\begin{array}{l}\text { Haben sich die Anforderungen } \\
\text { an die Qualifikation von Auf- } \\
\text { sichtsräten geändert? Wenn ja, } \\
\text { wie? }\end{array}$ & $\begin{array}{l}\text { Ja. Der Aufsichtsrat (Präsidium) nimmt } \\
\text { als Vertreter der Mitgliederversamm- } \\
\text { lung die Aufsicht über den Vorstand } \\
\text { wahr. Als satzungsmäßiges Kontrollor- } \\
\text { gan trägt er eine hohe Verantwortung. }\end{array}$ & $\begin{array}{l}\text { Das Geschäft ist schwieriger gewor- } \\
\text { den. Die unternehmerischen Risiken } \\
\text { sind größer, Fehlentscheidungen wir- } \\
\text { ken sich weitereichender aus. Deshalb } \\
\text { müssen Entscheidungen anders vorbe- } \\
\text { reitet sein und gründlicher bedacht } \\
\text { werden. Entsprechend verändern sich } \\
\text { die Anforderungen an Gremienmitglie- } \\
\text { der. Insbesondere juristische Kompe- } \\
\text { tenzen und Betriebswirtschaft sind } \\
\text { wichtiger geworden. Expertentum ist } \\
\text { eher von Nachteil, weil die Gefahr eines } \\
\text { „Schattenkabinetts“ gegenüber der } \\
\text { Führung besteht. Den Anforderungen } \\
\text { wird nicht durch zusätzliche Sitzungen, } \\
\text { sondern durch ausführliche, meist } \\
\text { ganztägige Sitzungen Rechnung getra- }\end{array}$ & $\begin{array}{l}\text { Ja, die Anforderungen haben sich sehr } \\
\text { verändert. Es sind mehr die geschäftli- } \\
\text { chen, kaufmännischen, unternehmeri- } \\
\text { schen Kompetenzen gefragt als die je- } \\
\text { weilige produktbezogene Fachlichkeit. } \\
\text { Auch politische Kontakte gehören heu- } \\
\text { te dazu. Statt „Gutmenschen“ werden } \\
\text { eher Leute gebraucht, die in der Lage } \\
\text { sind, die Aufsichts- und Kontrollfunkti- } \\
\text { on professionell, kompetent und sach- } \\
\text { verständig auszuüben. }\end{array}$ \\
\hline $\begin{array}{l}\text { Wie viel Zeit investieren Sie } \\
\text { pro Woche im Durchschnitt in } \\
\text { Ihr }\end{array}$ & $\begin{array}{l}\text { Früher (als Vorstand) mehrere Tage } \\
\text { pro Woche, heute im Schnitt zwei Tage } \\
\text { für Arbeit, Gremien und Repräsentati- }\end{array}$ & Etwa einen Tag pro Woche. & $\begin{array}{l}\text { Für beide Amter zusammen etwa einen } \\
\text { Tag pro Woche. }\end{array}$ \\
\hline $\begin{array}{l}\text { Ist es heute schwieriger als } \\
\text { früher, neue Mitglieder zu ge- } \\
\text { winnen? }\end{array}$ & $\begin{array}{l}\text { Nein. Wichtig ist aber die Mischung } \\
\text { aus Menschen im aktiven Berufsleben } \\
\text { und aus Pensionären. }\end{array}$ & $\begin{array}{l}\text { Nein. Für eine attraktive Aufgabe findet } \\
\text { man auch qualifizierte Leute. }\end{array}$ & $\begin{array}{l}\text { Nein. Beim Paritätischen Wohlfahrts- } \\
\text { verband gibt es ein natürliches Interes- } \\
\text { se der Mitglieds-Geschäftsführer, in } \\
\text { den Verbandsgremien mitzuwirken. }\end{array}$ \\
\hline $\begin{array}{l}\text { Wie ist das Zahlenverhältnis } \\
\text { von Frauen und Männern in } \\
\text { Ihrem Gremium? Sind Sie da- } \\
\text { mit zufrieden oder würden Sie } \\
\text { gerne etwas ändern? }\end{array}$ & Eine Frau und sechs Männer. & $\begin{array}{l}\text { Von zehn Mitgliedern des Aufsichtsra- } \\
\text { tes sind drei Frauen. }\end{array}$ & $\begin{array}{l}\text { Im Vorstand des Paritätischen Baden- } \\
\text { Württemberg sitzen drei Frauen und } \\
\text { acht Männer. Beim Baden-Württem- } \\
\text { bergischen Landesverband für Präven- } \\
\text { tion und Rehabilitation sind es eine } \\
\text { Frau und sechs Männer. }\end{array}$ \\
\hline $\begin{array}{l}\text { Welche Altersspanne halten } \\
\text { Sie für ein Aufsichtsgremium } \\
\text { für richtig? }\end{array}$ & $\begin{array}{l}\text { Die Satzung des Christlichen Jugend- } \\
\text { dorfwerks Deutschlands lässt eine } \\
\text { Wiederwahl bis zum 72. Lebensjahr zu. }\end{array}$ & $\begin{array}{l}\text { Der Altersschnitt im Aufsichtsrat liegt } \\
\text { derzeit über } 60 \text { Jahren. Eine Altersbe- } \\
\text { grenzung in Gremien wäre sinnvoll. }\end{array}$ & $\begin{array}{l}\text { Beim Paritätischen Baden-Württem- } \\
\text { berg ist ein Mitglied unter } 50 \text {, sieben } \\
\text { sind zwischen } 50 \text { und } 60 \text { und drei über } \\
60 \text { Jahre alt. Das ist im Prinzip eine } \\
\text { gute Mischung. Jüngere Mitglieder ha- } \\
\text { ben nicht kandidiert oder wurden nicht }\end{array}$ \\
\hline $\begin{array}{l}\text { Sollte man Ihrer Meinung nach } \\
\text { die Zahl der Amtsperioden von } \\
\text { Aufsichtsräten begrenzen? }\end{array}$ & Nein. & $\begin{array}{l}\text { Nein. Amtsperiode beträgt nach Sat- } \\
\text { zung fünf Jahre. Wahl oder Wiederwahl } \\
\text { ist nur vor dem } 70 \text {. Geburtstag mög- }\end{array}$ & $\begin{array}{l}\text { Nein zu einer Altersbegrenzung und } \\
\text { nein zu einer Amtszeitbegrenzung. }\end{array}$ \\
\hline $\begin{array}{l}\text { Was halten Sie von der Hono- } \\
\text { rierung von zeitaufwendigen } \\
\text { Ehrenämtern? }\end{array}$ & $\begin{array}{l}\text { Wer Verantwortung trägt und Zeit ein- } \\
\text { setzt, sollte angemessen honoriert } \\
\text { werden. }\end{array}$ & $\begin{array}{l}\text { Ein Sitzungsgeld für alle Gremienmit- } \\
\text { glieder und eine monatliche pauschale } \\
\text { Aufwandsentschädigung für die Gre- } \\
\text { mienmitglieder mit herausgehobener } \\
\text { Position, besonderem Zeiteinsatz und } \\
\text { besonderer Verantwortung sind in Ord- } \\
\text { nung („ordentliche Bezahlung“). }\end{array}$ & $\begin{array}{l}\text { Honorierung des Zeitaufwandes eher } \\
\text { nein, pauschale Aufwandsentschädi- } \\
\text { gung analog zur Praxis im Kommunal- } \\
\text { bereich ja. }\end{array}$ \\
\hline $\begin{array}{l}\text { Würden Sie Ihren Kindern } \\
\text { empfehlen, sich auf eine sol- } \\
\text { che Aufgabe einzulassen? }\end{array}$ & Ja. & Ja. & Ja. \\
\hline $\begin{array}{l}\text { Würden Ihre Kinder eine sol- } \\
\text { che Aufgabe übernehmen? }\end{array}$ & $\begin{array}{l}\text { Bisher alle stark im Beruf engagiert. } \\
\text { Kann ja noch kommen. }\end{array}$ & Ja. & Ja. \\
\hline
\end{tabular}


- innere Unabhängigkeit von den handelnden Personen

- wirtschaftliche Unabhängigkeit von der zu beaufsichtigenden Einrichtung

- Generalisten vor Spezialisten

- keine direkten Wettbewerber, aber gerne Fachleute aus der Branche

- keine Aufsichtsbehörden und keine aktiven Politiker, weil insbesondere auf der Kommunalebene Interessenkonflikte nicht zu vermeiden sind

- keine Lieferanten, für die das Unternehmen ein wichtiger Kunde ist

- keine Bankenvertreter, deren Institut Kredite an das Werk gegeben hat

- keine Verwandtschaftsgrade, weder innerhalb des Gremiums, noch zwischen Aufsicht und Leitung
- keine ehemaligen leitenden Mitarbeiter des Unternehmens

- nicht bezahlt, aber respektvoll honoriert

Aus den drei Vorsitzenden-Gesprächen lassen sich noch weitere Hinweise ableiten oder verstärken (vgl. Kasten unten). Sie eignen sich als Anregung oder als Aufreger für die nächste Aufsichtsratsklausur. Hohe Aufmerksamkeit und lebhafte Diskussion sind garantiert!

Zum Schluss sei noch bemerkt, dass der Autor - selber mehrfacher Aufsichts- und Stiftungsrat in der Sozialwirtschaft - den ehrenamtlichen Einsatz von Zeit und Kraft (manchmal auch von Geld oder Sachleistungen) hoch schätzt und würdigt. Es gibt kaum eine edlere Form des Engagements wie die, seine knappe Zeit und seinen reichen Erfahrungsschatz für eine gute Sache einzusetzen. Aber auch Aufsichtsräte sind keine Heiligen - und das ist gut so! Deshalb sei die Beschäftigung mit kritischen und selbstkritischen Themen dringend empfohlen.

\section{Vier Tipps zum Thema »Aufsichtsräte«}

\section{Gremien verkleinern!}

Je mehr Interessengruppen an der Arbeit Ihres Unternehmens Anteil nehmen (sprich: Einfluss nehmen wollen, ohne das unternehmerische Risiko mit zu übernehmen), desto höher ist der Druck auf Sitze im Aufsichtsgremium. Dieser Druck ist aber in der Regel ein Ausdruck für gruppenegoistische Interessen und nicht für Engagement für die gemeinsame Sache. Es gelten folgende Regeln:

- Je kleiner das Gremium, desto wichtiger das einzelne Mitglied!

- Je kleiner das Gremium, desto relevanter die Beteiligung!

- Je kleiner das Gremium, desto weniger Raum für Positionskämpfe und Eifersüchteleien!

2. Gremienmitglieder pauschaliert honorieren (Aufwandsentschädigung) und damit die Erwartung von Präsenz und Verfügbarkeit verbinden

- Bieten Sie dem oder den Vorsitzenden, die schließlich die Hauptarbeit machen, eine anständige Aufwandsentschädigung an. Alles pauschalieren! Keine kleinlichen Abrechnungsverpflichtungen!

- Erwarten Sie dann auch zu Recht von dem oder den Vorsitzenden einen Zeiteinsatz, der eine tatsächliche Kontrolle und Begleitung der Führungsebene möglich macht.

- Aufsichtsratsmitglieder, die eine besondere Aufgabe übernehmen (z. B. arbeitsintensiver Vorsitz eines Ausschusses) sollten ebenfalls pauschal honoriert werden.

- Alle übrigen Mitglieder, die nur zu den drei oder vier Sitzungen im Jahr antreten müssen, sollten nicht in Geld honoriert, sondern mit einem jährlichen gesellschaftlichen Event, zu dem die jeweiligen Partner eingeladen sind (Theaterbesuch, Schifffahrt, Wanderung) und so die ihnen zustehende Wertschätzung erfahren.

\section{Keine Proporzregelungen bei der Besetzung von Gremien}

Der Proporz ist die schwächste aller Auswahlmethoden. Proporz bedeutet immer: funktioniert nur in Schönwetterphasen. Wenn Sturm aufkommt und wenn es weh tut, dann ist Proporz mit Blockade gleich zu setzen.

4. Stellen Sie sich von Zeit zu Zeit im Aufsichtsgremium die selbstkritische Frage nach Ihrer Wirksamkeit und Ihrer Rollensicherheit!

Die Existenz des Gremiums wird zwar von der Satzung gesichert, aber trotzdem sei die Frage empfohlen, was das Gremium eigentlich bewirkt oder sichert oder verhindert. Fallen den Gremienmitgliedern bei einer Strategieklausur dazu nicht viele Punkte ein, dann ist entweder der Vorstand zu stark oder das Aufsichtsgremium zu schwach. Stellen Sie fest, dass das Gremium überwiegend als Bremser tätig ist, dann werden Sie bald mit einem Vorstandswechsel rechnen müssen. Fällt Ihnen auf, dass das Gremium laufend versucht, den Vorstand durch eigene Initiativen zu überholen, dann laufen Sie die gleiche Gefahr. Lassen sich Ihre Vorstände beide Formen aufsichtsrätlichen Fehlverhaltens klaglos gefallen, dann haben Sie die falschen Vorstände und sollten sich bald entsprechende Gedanken machen. 